\title{
Deep Brain Nuclei T1 Shortening after Gadobenate Dimeglumine in Children: Influence of Radiation and Chemotherapy
}

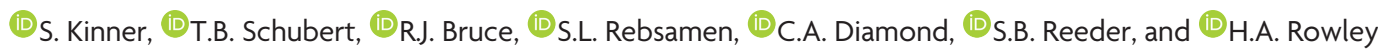

\begin{abstract}
BACKGROUND AND PURPOSE: Intrinsic T1-hyperintense signal has recently been reported in the deep gray nuclei on brain MR imaging after multiple doses of gadolinium-based contrast agents. Most reports have included adult patients and excluded those undergoing radiation or chemotherapy. We investigated whether $\mathrm{Tl}$ shortening is also observed in children and tried to determine whether radiochemotherapy is a risk factor for this phenomenon.
\end{abstract}

MATERIALS AND METHODS: In this single-center retrospective study, we reviewed clinical charts and images of all patients 18 years of age or younger with $\geq 4$ gadobenate dimeglumine-enhanced MRIs for 6 years. Seventy-six children (mean age, 9.3 years; 60 unconfounded by treatment, 16 with radiochemotherapy) met the selection criteria ( $>4$ MR imaging examinations; mean, 8 ). T1 signal intensity ratios for the dentate to pons and globus pallidus to thalamus were calculated and correlated with number of injections, time interval, and therapy.

RESULTS: Among the 60 children without radiochemotherapy, only 2 had elevated T1 signal intensity ratios ( $n=20$ and 16 injections). Twelve of the 16 children with radiochemotherapy showed elevated signal intensity ratios. Statistical analysis demonstrated a significant signal intensity ratio change for the number of injections $(P<.001)$ and amount of gadolinium $(P=.008)$, but not for the interscan time interval $(P=.35)$. There was a significant difference in the average signal intensity ratio change between those with and without radiochemotherapy $(P<.001)$. Chart review revealed no new neurologic deficits in any patients, related to their underlying conditions and prior surgeries.

CONCLUSIONS: Compared with published adult series, children show a similar pattern of $\mathrm{T1}$ hyperintense signal changes of the dentate and globus pallidus after multiple gadobenate dimeglumine injections. The T1 signal changes in children may have a later onset but are accelerated by radiochemotherapy.

ABBREVIATION: RCTX = radiochemotherapy

D uring the past year, several reports have been published describing the hyperintense appearance of the deep brain nuclei of adult patients on unenhanced T1-weighted MR images. This increased signal intensity occurred in patients with normal renal function who underwent multiple gadolinium-enhanced MR imaging examinations. ${ }^{1-13}$ This phenomenon has been evaluated using multiple different gadolinium-based contrast agents, including the linear agents gadodiamide, ${ }^{1-4}$ gadobenate

Received May 17, 2017; accepted after revision August 22.

From the Departments of Radiology (S.K., T.B.S., R.J.B., S.L.R., S.B.R., H.A.R.), Pediatrics (C.A.D.), Medical Physics (S.B.R.), Emergency Medicine (S.B.R.), Biomedical Engineering (S.B.R.), and Medicine (S.B.R.), University of Wisconsin-Madison, Madison, Wisconsin; Department of Diagnostic and Interventional Radiology and Neuroradiology (S.K.), University Hospital Essen, Essen, Germany; and Clinic of Radiology and Nuclear Medicine (T.B.S.), Basel University Hospital, Basel, Switzerland.

Please address correspondence to Sonja Kinner, MD, Hufelandstr 55, University Hospital Essen, Essen, Germany; e-mail: Sonja.Kinner@uni-due.de

http://dx.doi.org/10.3174/ajnr.A5453 dimeglumine, ${ }^{4,5}$ and gadopentetate dimeglumine $e^{6-10}$ as well as the macrocyclic agents gadoterate meglumine $e^{7,11}$ and gadobutrol. ${ }^{8,10,12,13}$ Postmortem studies have demonstrated the presence of gadolinium in these deep nuclei within the brain, confirming that increased signal intensity is likely due to the deposition of gadolinium. ${ }^{3,14,15}$ Recent animal studies have also shown this in rats. ${ }^{16-18}$

To date, most of the human studies have involved adult patients, with only 1 adult study including a small group of pediatric patients. ${ }^{6}$ Three recent studies analyzed smaller groups of up to 21 children undergoing gadopentetate dimeglumine examinations. ${ }^{19-21}$ In addition, 2 case reports described 3 pediatric patients with up to 35 contrast-enhanced MRIs, using gadopentetate dimeglumine. ${ }^{22,23}$ Similar to the adult population, these patients had multiple courses of chemotherapy, radiation therapy, and external beam radiation for orbital rhabdomyosarcoma. In fact, earlier reports of an increased signal of the dentate nucleus in 
unenhanced T1-weighted images attributed the changes to brain irradiation. $^{24}$ This study included only patients 30 years of age and older and did not analyze the number of contrast-enhanced MRIs performed. Studies on unconfounded adult patients are rare.

A very recent publication evaluated whether increased signal intensities can also be found after multiple injections of the macrocyclic agent gadoterate meglumine in children. ${ }^{25}$ As in all other pediatric patient studies, the authors explicitly excluded patients with a tumor in the cerebellum. Patients having had or undergoing chemotherapy were evaluated, but no subanalysis for differences was presented.

To date, pediatric studies are limited to investigations on the linear agent gadopentetate dimeglumine and the macrocyclic agent gadoterate meglumine. Therefore, the purpose of this retrospective study was 2-fold: 1) to investigate whether T1 shortening is also observed in children after multiple injections of gadobenate dimeglumine, and 2) to investigate whether brain radiation or chemotherapy or both impact this phenomenon.

\section{MATERIALS AND METHODS \\ Patients}

In this institutional review board-approved (University Hospital Essen), single-center retrospective study, we performed a search of the image data base to extract all patients 18 years of age and younger with at least 4 contrast-enhanced MRIs archived on a PACS between August 2009 and September 2015. We reviewed all clinical charts and images to verify that all patients received only 1 gadolinium agent (gadobenate dimeglumine, used routinely from this time point onward). The remaining patients were screened for treatment variables and underlying diseases known to potentially present with increased T1 signal in deep brain nuclei (like Langerhans cell histiocytosis and neurofibromatosis). To create comparable groups, we chose to evaluate 2 groups: patients with no radiation and/or chemotherapy (RCTX) (group 1) and those with a posterior fossa tumor undergoing RCTX (group 2). Figure 1 represents a flow chart showing the patient selection. Patients with large, bilateral posterior fossa tumors involving or masking the dentate nucleus on both sides were excluded because these nuclei would be difficult or impossible to evaluate on preoperative imaging. In unilateral tumors, the dentate nucleus of the contralateral side was evaluated.

For all patients included in the study, age, sex, and diagnoses were documented. We also reviewed the electronic health records for any new or unexplained onset of movement disorders or cerebellar dysfunction. In the patients with posterior fossa tumors, whether they had surgery and which chemotherapy and radiation therapy they received were also assessed. If patients received radiation therapy, it was always a tumor-selective radiation therapy plan. In addition, the time interval between the first and last MR imaging was documented as well as the total cumulative amount of gadolinium injected across time.

\section{MR Imaging Protocol}

MR imaging of the brain was performed on clinical 1.5T (Optima MR450w or Signa HDxt; GE Healthcare, Milwaukee, Wisconsin)

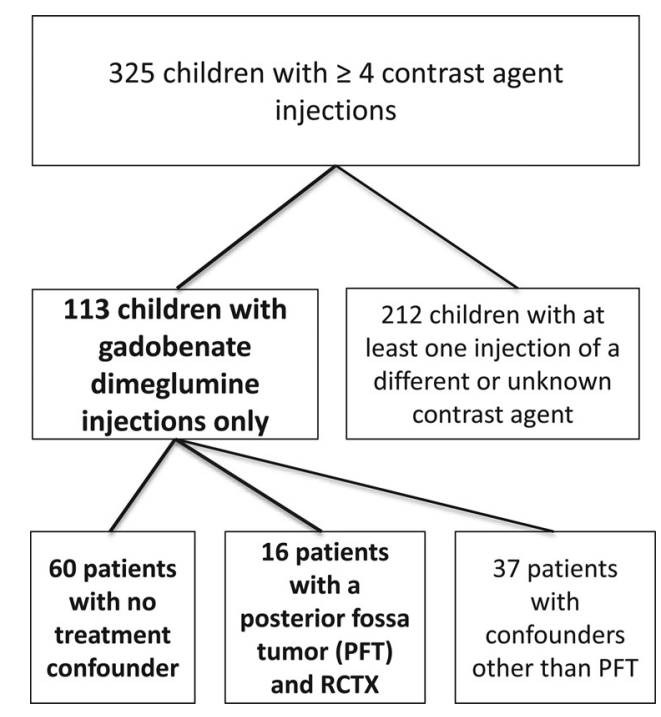

FIG 1. Flow chart showing the patient-selection criteria. PFT indicates posterior fossa tumor.

or 3T scanners (Discovery MR750 or MR750w; GE Healthcare) using 8-channel head coils. Imaging protocols included the following sequences before administration of gadobenate dimeglumine at a dosage of $0.1 \mathrm{mmol}(0.2 \mathrm{~mL})$ per kilogram of body weight: axial T1-weighted 3D inversion recovery-prepared fastspoiled gradient-echo sequences (BRAVO: section thickness, 3 $\mathrm{mm}$; TR, 8.5-9.3ms; TE, 3.2-3.7ms; flip angle, $13^{\circ}[1.5 \mathrm{~T}], 12^{\circ}$ [3T]; TI, $450 \mathrm{~ms}$ ) and/or sagittal T1-weighted 2D fluid-attenuated inversion recovery (section thickness, $3 \mathrm{~mm}$; TR, $2212 \mathrm{~ms}$ [1.5T], 3050-3400 ms [3T]; TE, 8.4-8.8 ms [1.5T], 23.3-23.9 ms [3T]; flip angle, $90^{\circ}[1.5 \mathrm{~T}], 111^{\circ}[3 \mathrm{~T}]$; TI, $750 \mathrm{~ms}$ [1.5T], 917-964 ms [3T]).

\section{Image and Data Analysis}

As a first step, visual analysis of the first and last MR imaging was performed, and a qualitative visual signal increase (present or not present) was documented. For objective image analysis, we analyzed images as previously described by Kanda et $\mathrm{al}^{26}$ : De-identified images were imported and subsequently analyzed with open-source Digital Imaging and Communications in Medicine software OsiriX MD (Version 2.5.1 64-bit; http:// www.osirix-viewer.com). Image analysis was performed by a radiologist with 10 years' experience in MR imaging and specific experience in neuroradiology and pediatric imaging. A freehand ROI was drawn on the unenhanced T1-weighted images in the bilateral dentate nuclei, the central pons, the middle cerebellar peduncle, and the left and right globus pallidus as well as the left and right thalamus, excluding the pulvinar thalami, which have been shown to exhibit $\mathrm{T} 1$ shortening as well in patients after multiple linear gadolinium-based contrast agent injections. To ensure accurate segmentation of the dentate nucleus, we performed correlation with $\mathrm{T} 2$ - and $\mathrm{T} 22^{*}$-weighted images when necessary. We also added the middle cerebellar peduncle as a correlation in addition to the pons, as described by Ramalho et al. ${ }^{4}$ We therefore assessed not only the dentateto-pons ratios but also the dentate-to-middle cerebellar peduncle ratios. Dentate-to-pons, dentate-to-middle cerebellar 


\begin{tabular}{lccc}
\hline & Unconfounded Cases & Posterior Fossa Tumors & $P$ \\
\hline Total No. of patients & 60 & 16 & $7.8(4-10)$ \\
Age $(y r)$ & $8.7(6-14)$ & $6(37.5 \%)$ & .095 \\
Sex: female & $30(50.0 \%)$ & $14.5(11.2-16)$ & .543 \\
Contrast-enhanced MRIs & $7(5-9)$ & $3.4(1.4-4.7)$ & $<.001$ \\
First-to-last MRI with contrast $(y r)$ & $3.1(1.3-4.7)$ & $63.8(49.8-130.5)$ & .794 \\
Accumulated dose of gadoterate dimeglumine $(\mathrm{mL})$ & $48.5(31.1-87.2)$ & $0.090(0.049-0.132)$ & .161 \\
Mean ratio change of dentate to pons & $0.028(0.007-0.049)$ & $0.130(0.091-0.168)$ & $<.001$ \\
Mean ratio change of globus pallidus to thalamus & $0.034(0.013-0.055)$ & $<.001$ \\
\hline
\end{tabular}

Note:-min indicates minimum; max, maximum.

${ }^{a}$ Data are presented as means with $95 \%$ confidence intervals in parentheses.

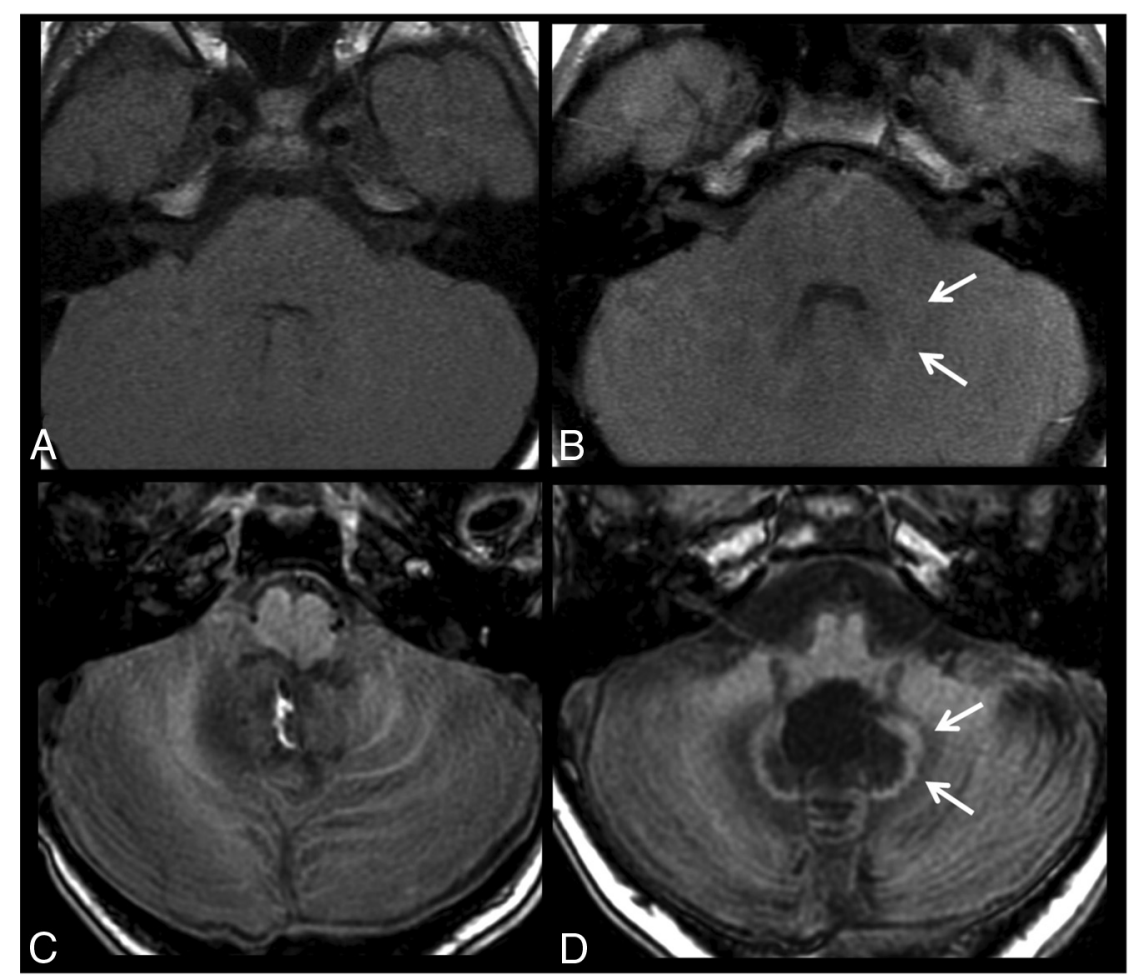

FIG 2. T1-weighted images acquired before $(A$ and $C)$ and after 20 gadobenate injections $(B$ and $D)$ in a patient with follow-up for optic glioma without radiation or chemotherapy $(A$ and $B)$ and a patient with medulloblastoma after an operation and radiochemotherapy $(C$ and $D)$. Subtle signal changes of the dentate (arrows) can be seen in the patient without any therapy, while the patient with RCTX shows distinct Tl signal changes of the dentate and perifocal edema.

peduncle, and globus pallidus-to-thalamus ratios were calculated from the first and last MR imaging dataset. The ratio change between the first and last imaging was calculated.

\section{Statistical Analysis}

Statistical analysis was performed with R statistical and computing software (http://www.r-project.org). Intraclass correlation coefficients $(2,1)$ were used to assess the reliability of the ratio measurements between the means of the cerebellar peduncle and pons.

Mixed-effects linear regression was used to test the ratio between different factors (number of injections, time between first and last imaging, and amount of gadolinium injected) with subject as a random effect. Repeated-measures ANOVA was used to test whether the change in the ratio across time was significantly different between factor groups with subject as a random effect. Pearson correlation coefficients were calculated to assess the linear relationship between total gadolinium dos- age and signal intensity ratio change for patients with and without therapy.

\section{RESULTS}

A total of 325 children with at least 4 contrast injections were identified. Of these, 76 children (mean age, 9.3 years) met the listed selection criteria (4-20 contrast-enhanced MRIs; mean, 8). Sixteen patients had posterior fossa tumors treated with radiation alone $(n=2)$, combined chemo- and radiation therapy $(n=9)$, and chemotherapy alone $(n=5)$, and 60 were unconfounded by such treatment. The characteristics of both patient groups can be found in the Table.

On visual inspection, 12 of the 16 patients with posterior fossa tumor and radiation or chemotherapy showed hyperintensities of the deep brain nuclei. These patients had undergone between 10 and 20 contrast injections, and the 4 patients without signal increase had $<10$ gadobenate dimeglumine injections. In the 60 patients without RCTX, only 2 patients were found to have a visual signal increase of the dentate nucleus or the globus pallidus between the first and last brain MR imaging. These patients had the highest contrast exposures, with 20 and 16 gadobenate dimeglumine injections.

Figure 2 shows a comparison of 2 patients after 20 injections, one with no treatment variables and 1 patient with medulloblastoma after radiochemotherapy. Figure 3 shows images before and after 10 gadobenate dimeglumine injections in a patient with no treatment variables and a second patient with a medulloblastoma, after surgery and radiochemotherapy.

Statistical analysis showed a statistically significant change in the signal ratio for the number of scans and contrast injections $(P<.001)$ as well as the amount of gadolinium $(P=.008)$, but not for the interscan time interval $(P=.353)$. For each additional contrast-enhanced MR imaging, the signal ratio increased by 0.01 on average. There was a significant difference in the average change in ratio with time between those with RCTX versus those without $(P<.001)$. A comparison of the ratio change for the 


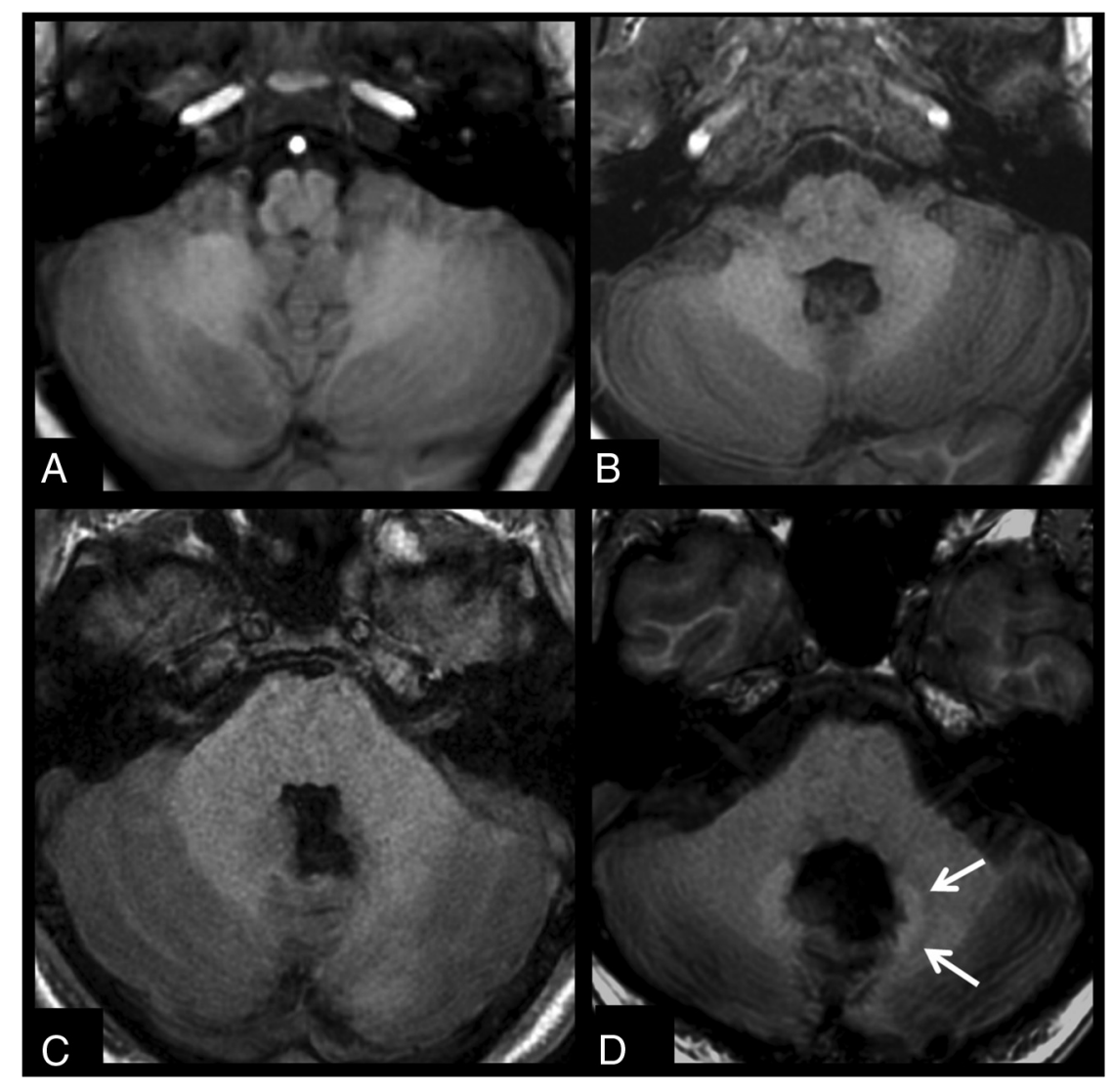

FIG 3. T1-weighted images acquired before $(A$ and $C$ ) and after 10 gadobenate injections ( $B$ and $D$ ) in a patient being followed for a mass at the craniocervical junction with only surgical therapy $(A$ and $B$ ) and a patient with medulloblastoma after an operation and radiochemotherapy ( $C$ and $D$ ). No signal change can be seen in the dentate for the patient without RCTX, while the patient with RCTX already shows signal changes after 10 injections.

dentate to pons and globus pallidus to thalamus showed no significant difference in average change in the ratio between measurements in the dentate and in the globus pallidus $(P=.199)$, like that of children after focal radiation of the cerebellum $(P=$ .4). Furthermore, no statistically significant difference was found for the use of the middle cerebellar peduncle or the pons as a control for the dentate $(P=.39)$.

Figure 4 shows a distribution of the signal ratio change for the globus pallidus compared with thalamus in children with no treatment variables and children with radiochemotherapy.

Figure 5 shows the correlation of the total gadolinium dose administered and the signal ratio change for patients with and without therapy.

Directed neurologic chart review revealed no obvious new neurologic clinical deficits in the children with apparent T1 hyperintensities. We specifically searched for signs or symptoms referable to the extrapyramidal motor system such as tremor, bradykinesia, movement disorders, and motor dysfunction, which were not explained by the patient's known disease process.

\section{DISCUSSION}

In this retrospective study, we investigated whether pediatric patients show T1 shortening of deep brain nuclei after multiple doses of gadobenate dimeglumine and whether there is any difference between patients with and without radiation and/or chemotherapy.
This study has 4 messages we believe to be important:

First, children show a pattern of $\mathrm{T} 1$ signal changes of the dentate nucleus and globus pallidus after multiple injections of gadobenate dimeglumine, like that reported in adults. This has been shown for gadopentetate dimeglumine in a few recent small studies in children. ${ }^{19-21}$ We were able to show it for gadobenate dimeglumine, another linear contrast agent, in a pediatric patient cohort for the first time. A first study in pediatric patients examining the effect of multiple doses of a macrocyclic contrast agent supports similar results in adults: Macrocyclic agents show no signal intensity increase after serial injections of a macrocyclic agent. ${ }^{7,8,12,27}$ The study by Stojanov et al, ${ }^{13}$ reporting a signal increase for a macrocyclic agent has been severely criticized because visual inspection of reported images did not seem to support the quantitative results of the study.

The second key finding is that patients without radiochemotherapy were much less likely to show T1 changes. In the absence of RCTX, only 2/60 showed these changes, and these occurred in the 2 patients with the most gadobenate dimeglumine injections ( $n=16$ and 20, respectively). This observation is consistent with findings in adults for gadobenate dimeglumine: Ramalho et $\mathrm{al}^{4}$ investigated the influence of 2 linear contrast agents (gadodiamide and gadobenate dimeglumine) on the dentate nucleus and globus pallidus and showed a significant signal increase with gadodiamide-enhanced studies, but not with gadobenate dimeglumine. Their adult patient population comprised only patients without prior targeted or whole-brain radiation and those without multiple sclerosis and other diseases that might result in T1 shortening of deep brain nuclei and can thus be considered unconfounded like our 60-patient subgroup. However, the maximal number of MR images obtained in their patient population was 11 examinations. Because we saw an increase only after 16 examinations in unconfounded children, the number of injections might be too small in the study of Ramalho et al to find a visually discernible effect. Weberling et al, ${ }^{5}$ however, investigated adult patients with melanoma with 5-15 injections of gadobenate dimeglumine (on average, 7.7 injections) and found an increase in signal intensity in the dentate nucleus. The results of Weberling et al in adults are therefore in line with ours in children: There seem to be a certain number of injections needed before signal intensity changes become visible. They, like Adin et al ${ }^{6}$ and Radbruch et al, ${ }^{7}$ did not find a correlation between radiation therapy and signal changes in adults, which is different from our results in children. 


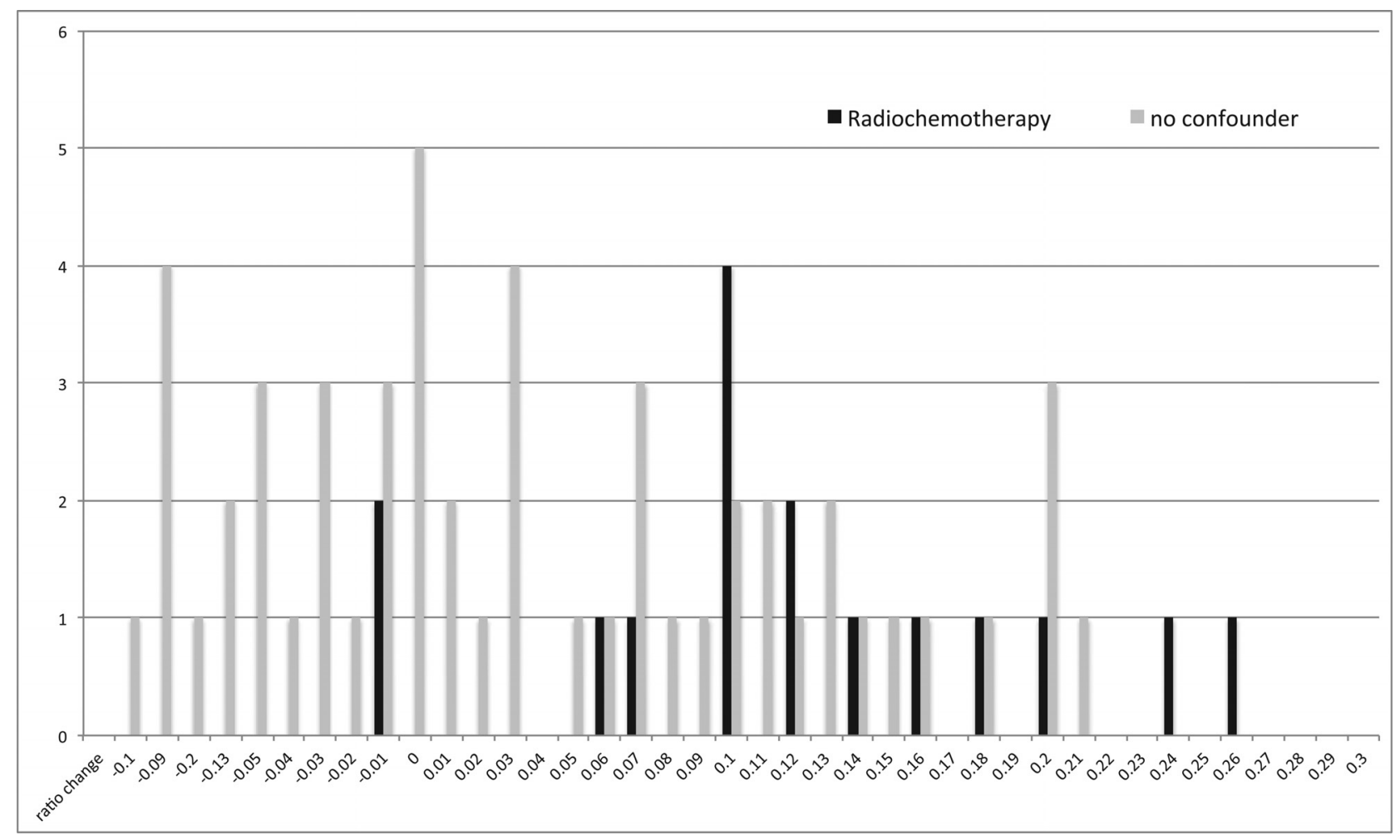

FIG 4. Distribution of the signal ratio change of the globus pallidus compared with the thalamus (globus pallidus to thalamus) in children with no treatment variables (gray bars) and children with radiochemotherapy (black bars) shows a shift/tendency to higher ratio changes in children with posterior fossa tumors under therapy.

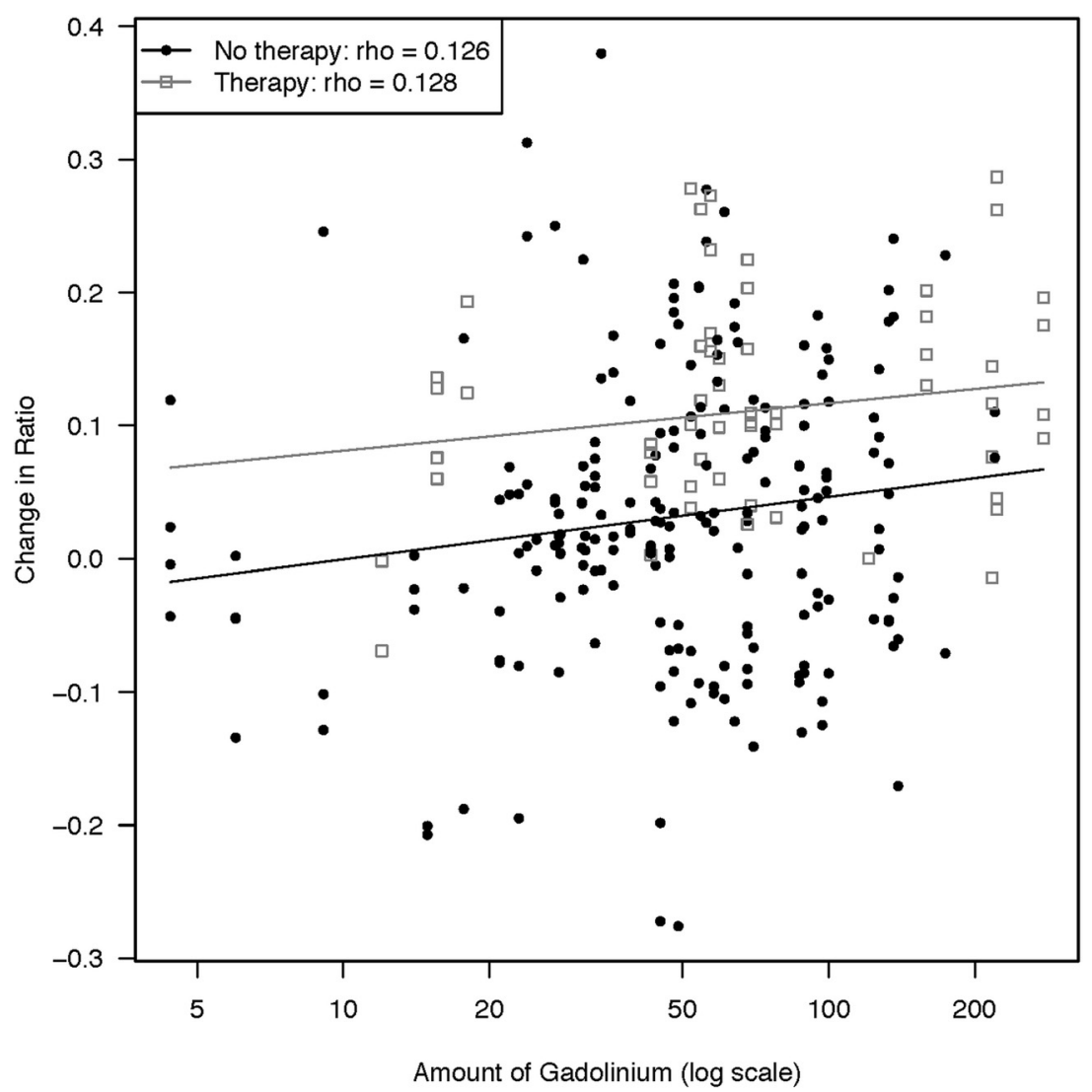

FIG 5. Scatterplot showing the correlation between cumulative gadolinium doses and signal ratio change for patients with and without therapy.
We also found that the time interval from gadolinium-based contrast agent injection until signal changes occur in children can be significantly reduced in the setting of radiochemotherapy, which impacts the blood-brain barrier. While Weberling et $\mathrm{al}^{5}$ did not find a correlation for radiation therapy, they did not test for differences concerning chemotherapy. Because most of their adult patients had melanoma, it can be assumed that many patients received chemotherapy, even if not explicitly stated. This is important because we found that in patients receiving radiation therapy for posterior fossa tumors, the signal of the dentate changed like the signal of the globus pallidus, which leads to the presumption that radiation therapy itself locally does not lead to signal changes or acceleration of these changes, but rather the therapy (radiation therapy and/or chemotherapy) has an influence on the blood-brain barrier, which leads to uptake of gadolinium in any of the deep brain nuclei.

Montagne et $\mathrm{al}^{28}$ showed, in a recent investigation, that blood-brain barrier breakdown is an early event in the aging human brain, starting from the hippocampus but also involving other brain 
regions. The fact that children who did not undergo radiation or chemotherapy showed a later onset of signal increase might be also due to their still fully intact blood-brain barrier.

A key message that can be drawn from this study that is also in accordance with other studies is that the observed signal changes are apparently asymptomatic and are also independent of the time between injections. In all patients presented in prior studies, as in our patient group 2, presumed gadolinium deposition has not been linked to clinical deficits but does appear to be an imagingobserved adverse effect of therapy. Although neurologically asymptomatic, the appearance is dependent on the number of injections and the cumulative amount of gadolinium. Therefore, until more follow-up studies and prospective neurologic and neurocognitive surveillance are available, each injection of a gadolinium-based contrast agent should be carefully considered. Other contrast agents, like iron oxide particles, can be a possible alternate to gadolinium-based agents in brain imaging. ${ }^{29,30}$

During this analysis, we incidentally found that the red nucleus shows an intensity change like that of the dentate and globus pallidus. This finding might suggest the deposition of gadolinium in the red nucleus as well. New onset of tremor, gait disturbances, or other neurologic deficits have not been found in these children. Further analysis will show how signal changes in the dentate nucleus and globus pallidus might correlate with signal changes in other deep brain nuclei, especially the red nuclei, all of which are also known to take up iron as a normal physiologic finding, so changes might be difficult to analyze and attribute to a certain origin.

The linear gadolinium agent we studied here was originally chosen and continues to be used in our practice due to the combination of proved beneficial T1 enhancing effects and an excellent risk profile. Gadobenate has the highest relaxivity of any of the gadolinium agents marketed for central nervous system applications. It is been shown in multiple double-blind randomized crossover studies to show better tumor-enhancing performance metrics compared with any of the other agents. ${ }^{31}$ It also has an excellent risk-benefit profile for nephrogenic systemic fibrosis, with thousands of renally impaired, high-risk patients given gadobenate due to diagnostic need in potentially life-threatening conditions, with no subsequent nephrogenic systemic fibrosis observed. ${ }^{32}$ At the present time, we are therefore continuing to use this agent in our practice, while still recognizing the potential for apparently asymptomatic T1 shortening and presumably gadolinium deposition in the deep gray nuclei.

Our study clearly has limitations: First, we cannot prove that the contrast agent we used, gadobenate dimeglumine, is the reason for the later onset of signal changes in children compared with adults in the literature. Direct comparisons with adults from our data base are forthcoming. In addition, we cannot exclude gadolinium being deposited in the brain regions we used as comparators in children because we do not yet have postmortem studies in children. If this is the case, the postulated later onset in children might be due to different storage amounts. Furthermore, postmortem studies in children are especially needed.

Also, we cannot exclude the T1-weighted sequences we used having an influence on the detectability rate of signal changes. In a letter, Kanda et $\mathrm{al}^{33}$ discussed different pulse sequences having different imaging appearances and different signal-to-noise ra- tios, which can have an influence on the image appearance and contrasts. We used conventional unenhanced T1-weighted gradient-echo sequences, which have been used in prior studies on this topic by different authors. ${ }^{3,5,12}$ Another issue is that different magnetic field strengths were used in these patients. However, Adin et $\mathrm{al}^{6}$ examined the influence of different field strengths in a subanalysis of their study and could show that no statistically significant difference was present.

\section{CONCLUSIONS}

Our study shows that children show a pattern of T1 signal changes of the dentate and globus pallidus after multiple injections of gadobenate dimeglumine like that in adults in published studies of this agent. The appearance in children may have a later onset and seems to be accelerated by radiation and chemotherapy.

Disclosures: Scott B. Reeder—UNRELATED: Grant: Bracco Diagnostics, Comments: provides an unrestricted grant for educational and research purposes*; Other: GE Healthcare, Comments: provides resources for research (but no money) to the University of Wisconsin*; Grants/Grants Pending: National Institutes of Health*; Stock/ Stock Options: Elucent Medical, Cellectar Biosciences, Calimetrix, Comments: I hold shares/ownership in Elucent Medical and Collectar Biosciences and am a founder of Calimetrix.* Howard A. Rowley-UNRELATED: Grant: Bracco Diagnostics, Comments: fellowship funding support in a department (not in my section)*; Consulting Fee or Honorarium: Bracco Diagnostics, Guerbet, Bayer, GE Healthcare, Comments: These are all companies that make gadolinium contrast that have provided me with honoraria or consulting fees, directly or indirectly, in the past 3 years; Support for Travel to Meetings for the Study or Other Purposes: Bracco Diagnostics; Consultancy: GE Healthcare, Comments: consulting related to regulatory matters; Payment for Lectures Including Service on Speakers Bureaus: Bracco Diagnostics, Comments: accredited lectures on several topics, including MR physics, dementia, and other subject areas; Patents (Planned, Pending or Issued): GE Healthcare, Comments: joint patent on an MRI pulse sequence, accepted by the Wisconsin patent office and pending issuance, not related to present work. *Money paid to the institution.

\section{REFERENCES}

1. Errante Y, Cirimele V, Mallio CA, et al. Progressive increase of T1 signal intensity of the dentate nucleus on unenhanced magnetic resonance images is associated with cumulative doses of intravenously administered gadodiamide in patients with normal renal function, suggesting dechelation. Invest Radiol 2014;49:685-90 CrossRef Medline

2. Quattrocchi CC, Mallio CA, Errante Y, et al. Gadodiamide and dentate nucleus $\mathrm{T} 1$ hyperintensity in patients with meningioma evaluated by multiple follow-up contrast-enhanced magnetic resonance examinations with no systemic interval therapy. Invest Radiol 2015; 50:470-72 CrossRef Medline

3. McDonald RJ, McDonald JS, Kallmes DF, et al. Intracranial gadolinium deposition after contrast-enhanced MR imaging. Radiology 2015;275:772-82 CrossRef Medline

4. Ramalho J, Castillo M, AlObaidy M, et al. High signal intensity in globus pallidus and dentate nucleus on unenhanced T1-weighted MR images: evaluation of two linear gadolinium-based contrast agents. Radiology 2015;276:836-44 CrossRef Medline

5. Weberling LD, Kieslich PJ, Kickingereder P, et al. Increased signal intensity in the dentate nucleus on unenhanced T1-weighted images after gadobenate dimeglumine administration. Invest Radiol 2015;50:743-48 CrossRef Medline

6. Adin ME, Kleinberg L, Vaidya D, et al. Hyperintense dentate nuclei on T1-weighted MRI: relation to repeat gadolinium administration. AJNR Am J Neuroradiol 2015;36:1859-65 CrossRef Medline

7. Radbruch A, Weberling LD, Kieslich PJ, et al. Gadolinium retention in the dentate nucleus and globus pallidus is dependent on the class of contrast agent. Radiology 2015;275:783-91 CrossRef Medline

8. Cao $Y$, Huang DQ, Shih G, et al. Signal change in the dentate nucleus on T1-weighted MR images after multiple administrations of gado- 
pentetate dimeglumine versus gadobutrol. AJR Am J Roentgenol 2015;206:414-19 CrossRef Medline

9. Zhang Y, Cao Y, Shih GL, et al. Extent of signal hyperintensity on unenhanced T1-weighted brain MR images after more than 35 administrations of linear gadolinium-based contrast agents. Radiology 2017;282:516-25 CrossRef Medline

10. Schlemm L, Chien C, Bellmann-Strobl J, et al. Gadopentetate but not gadobutrol accumulates in the dentate nucleus of multiple sclerosis patients. Mult Scler 2017;23:963-72 CrossRef Medline

11. Eisele P, Alonso A, Szabo K, et al. Lack of increased signal intensity in the dentate nucleus after repeated administration of a macrocyclic contrast agent in multiple sclerosis: an observational study. Medicine 2016;95:e4624 CrossRef Medline

12. Radbruch A, Weberling LD, Kieslich PJ, et al. High-signal intensity in the dentate nucleus and globus pallidus on unenhanced T1weighted images: evaluation of the macrocyclic gadolinium-based contrast agent gadobutrol. Invest Radiol 2015;50:805-10 CrossRef Medline

13. Stojanov D, Aracki-Trenkic A, Vojinovic S, et al. Increasing signal intensity within the dentate nucleus and globus pallidus on unenhanced T1W magnetic resonance images in patients with relapsingremitting multiple sclerosis: correlation with cumulative dose of a macrocyclic gadolinium-based contrast agent, gadobutrol. Eur Radiol 2016;26:807-15 CrossRef Medline

14. Kanda T, Fukusato T, Matsuda M, et al. Gadolinium-based contrast agent accumulates in the brain even in subjects without severe renal dysfunction: evaluation of autopsy brain specimens with inductively coupled plasma mass spectroscopy. Radiology 2015;276: 228-32 CrossRef Medline

15. Murata N, Gonzalez-Cuyar LF, Murata K, et al. Macrocyclic and other non-group 1 gadolinium contrast agents deposit low levels of gadolinium in brain and bone tissue: preliminary results from 9 patients with normal renal function. Invest Radiol 2016;51:447-53 CrossRef Medline

16. Robert P, Lehericy S, Grand S, et al. T1-weighted hypersignal in the deep cerebellar nuclei after repeated administrations of gadolinium-based contrast agents in healthy rats: difference between linear and macrocyclic agents. Invest Radiol 2015;50:473-80 CrossRef Medline

17. Frenzel T, Apte C, Jost G, et al. Quantification and assessment of the chemical form of residual gadolinium in the brain after repeated administration of gadolinium-based contrast agents: comparative study in rats. Invest Radiol 2017;52:396-404 CrossRef Medline

18. Jost G, Lenhard DC, Sieber MA, et al. Signal increase on unenhanced T1-weighted images in the rat brain after repeated, extended doses of gadolinium-based contrast agents: comparison of linear and macrocyclic agents. Invest Radiol 2016;51:83-89 CrossRef Medline

19. Hu HH, Pokorney A, Towbin RB, et al. Increased signal intensities in the dentate nucleus and globus pallidus on unenhanced T1-weighted images: evidence in children undergoing multiple gadolinium MRI exams. Pediatr Radiol 2016;46:1590-98 CrossRef Medline

20. Flood TF, Stence NV, Maloney JA, et al. Pediatric brain: repeated exposure to linear gadolinium-based contrast material is associated with increased signal intensity at unenhanced T1-weighted MR imaging. Radiology 2017;282:222-28 CrossRef Medline

21. Roberts DR, Chatterjee AR, Yazdani M, et al. Pediatric patients demonstrate progressive T1-weighted hyperintensity in the dentate nucleus following multiple doses of gadolinium-based contrast agent. AJNR Am J Neuroradiol 2016;37:2340 - 47 CrossRef Medline

22. Roberts DR, Holden KR. Progressive increase of T1 signal intensity in the dentate nucleus and globus pallidus on unenhanced T1weighted MR images in the pediatric brain exposed to multiple doses of gadolinium contrast. Brain Dev 2016;38:331-36 CrossRef Medline

23. Miller $\mathrm{JH}, \mathrm{Hu} \mathrm{HH}$, Pokorney A, et al. MRI brain signal intensity changes of a child during the course of $\mathbf{3 5}$ gadolinium contrast examinations. Pediatrics 2015;136:e1637-40 CrossRef Medline

24. Kasahara S, Miki Y, Kanagaki M, et al. Hyperintense dentate nucleus on unenhanced T1-weighted MR images is associated with a history of brain irradiation. Radiology 2011;258:222-28 CrossRef Medline

25. Radbruch A, Haase R, Kickingereder P, et al. Pediatric brain: no increased signal intensity in the dentate nucleus on unenhanced T1-weighted MR images after consecutive exposure to a macrocyclic gadolinium-based contrast agent. Radiology 2017;283:828-36 CrossRef Medline

26. Kanda $\mathrm{T}$, Ishii $\mathrm{K}$, Kawaguchi $\mathrm{H}$, et al. High signal intensity in the dentate nucleus and globus pallidus on unenhanced T1-weighted MR images: relationship with increasing cumulative dose of a gadolinium-based contrast material. Radiology 2014;270:834-41 CrossRef Medline

27. Kanda $\mathrm{T}$, Osawa $\mathrm{M}$, Oba $\mathrm{H}$, et al. High signal intensity in dentate nucleus on unenhanced T1-weighted MR images: association with linear versus macrocyclic gadolinium chelate administration. $R a$ diology 2015;275:803-09 CrossRef Medline

28. Montagne A, Barnes SR, Sweeney MD, et al. Blood-brain barrier breakdown in the aging human hippocampus. Neuron 2015;85: 296-302 CrossRef Medline

29. Corot C, Petry KG, Trivedi R, et al. Macrophage imaging in central nervous system and in carotid atherosclerotic plaque using ultrasmall superparamagnetic iron oxide in magnetic resonance imaging. Invest Radiol 2004;39:619-25 CrossRef Medline

30. Doyle KP, Quach LN, Arceuil HE, et al. Ferumoxytol administration does not alter infarct volume or the inflammatory response to stroke in mice. Neurosci Lett 2015;584:236-40 CrossRef Medline

31. Kanal E, Maravilla K, Rowley HA. Gadolinium contrast agents for CNS imaging: current concepts and clinical evidence. AJNR Am J Neuroradiol 2014;35:2215-26 CrossRef Medline

32. Bruce R, Wentland AL, Haemel AK, et al. Incidence of nephrogenic systemic fibrosis using gadobenate dimeglumine in 1423 patients with renal insufficiency compared with gadodiamide. Invest Radiol 2016;51:701-05 CrossRef Medline

33. Kanda $\mathrm{T}$, Oba $\mathrm{H}$, Toyoda $\mathrm{K}$, et al. Recent advances in understanding gadolinium retention in the brain. AJNR Am J Neuroradiol 2016;37: E1-2 CrossRef Medline 